\title{
Risques et menaces de l'Asie du Sud
}

\section{Christiane Hurtig}

\section{(2) OpenEdition \\ Journals}

Édition électronique

URL : http://journals.openedition.org/conflits/97

DOI : $10.4000 /$ conflits. 97

ISSN : $1777-5345$

Éditeur :

CCLS - Centre d'études sur les conflits lilberté et sécurité, L'Harmattan

Édition imprimée

Date de publication : 17 mai 1991

ISSN : 1157-996X

Référence électronique

Christiane Hurtig, « Risques et menaces de l'Asie du Sud », Cultures \& Conflits [En ligne], 02 I printemps 1991, mis en ligne le 30 décembre 2002, consulté le 30 mars 2021. URL : http:// journals.openedition.org/conflits/97 ; DOI : https://doi.org/10.4000/conflits.97

Ce document a été généré automatiquement le 30 mars 2021.

Creative Commons License 


\title{
Risques et menaces de l'Asie du Sud
}

\author{
Christiane Hurtig
}

1 A supposer que la crise et la guerre du Golfe aient pu se produire lus tôt avec leurs caractéristiques actuelles, on aurait sans doute vu l'Inde et le Pakistan réagir autrement qu'ils ne l'ont fait. Le gouvernement de Rajiv Gandhi aurait commencé par s'abstenir à l'ONU puis, une fois les opérations commencées, prendre parti pour l'Irak au nom de ses anciennes relations avec un pays non-aligné laïc qui avait toujours soutenu l'Inde dans le différend indo-pakistanais sur le Cachemire. Par opposition, le Pakistan du général Zia, fidèle allié des Etats-Unis dont les armées ont longtemps contribué à la sécurité interne de l'Arabie Saoudite et ont continué à assister les petits pays du Golfe, se serait peut-être plus clairement engagé au sein de la coalition.

2 Le fait même que les avions américains en provenance des que l'Inde considérait Philippines ou de la base de Diego Garcia (comme une menace directe depuis vingt ans) aient pu au début de la crise se ravitailler à Bombay, Agra et Madras, plutôt qu'à Karachi en dit long sur l'évolution de l'Asie du Sud comme sur la manière dont elle est perçue par les pays du Nord.

3 Cependant les positions ne se sont pas complètement retournées et la guerre, en révélant et accusant les contradictions internes aux pays en cause, a aussi freiné certaines remises en question. Ainsi le potentiel de menace de l'Asie du Sud, dont la guerre a occulté certains aspects en en soulignant d'autres, est devenu de plus en plus difficile à apprécier. Car il avait déjà été modifié, dans la dernière décennie par l'émergence de variables sociales nouvelles et on ne peut prédire comment l'instabilité qu'elles ont apportée s articulera avec les retombées politiques internes des craintes (notamment écologiques) et des débats engendrés tant par la guerre elle-même que par son issue rapide et, là-bas, inattendue.

On peut néanmoins avancer à titre d'hypothèse globale que les risques d'anomie sociale et de développements politiques chaotiques susceptibles de faire tache d'huile l'emportent sur les capacités de nuisance proprement militaires des différents pays de la région. Bien que le niveau d'armement de pays comme l'Inde et le Pakistan(1), le fait que le Pakistan consacre $6 \%$ de son PNB aux dépenses militaires (pour $4 \%$ en Inde, en années de pointe) et la position indienne de premier acheteur d'armes du monde, 
obligent à prendre leurs cas en considération, ni leur histoire ni les bases économiques de leur puissance ou leurs projets publics ne semblent les prédisposer à lancer des défis de type irakien. État-client des États-Unis, le Pakistan est longtemps demeuré sous contrôle. Plus autonome, l'Inde a fait du règlement pacifique des différends sa carte de visite et, tout en adaptant ses principes aux intérêts stratégiques d'une défense conçue comme incluant le contrôle de ses marches, elle ne s'en est jamais vraiment écartée qu'au profit d'autres traits de son identité(2). Les principales menaces que l'Asie du Sud pourrait faire peser sur l'ordre international ne sont pas militaires. Elles résulteraient plutôt de subversions internes débouchant sur une déstabilisation régionale qui, de proche en proche, pourraient affecter le Nord.

5 Autrefois les risques d'aventure entre Inde et Pakistan étaient liés aux vieilles rivalités entre ces frères siamois et à situer dans le contexte plus général de la traditionnelle défiance entre l'Inde et la Chine. Mais ce qui, il y a quelques années encore, pouvait inquiéter en raison de la puissance d'une Inde proche de l'Union soviétique et militant en faveur d'un nouvel ordre économique mondial, a laissé place, avec l'effacement de Moscou, à un ensemble de facteurs déséquilibrants que la guerre du Golfe a aggravé.

Le nouveau déséquilibre du système international interdit $d$ écarter absolument des risques de bouleversement politico-culturels qui mèneraient l'Inde et le Pakistan sur la voie des provocations. Déjà le danger de les voir opter pour une véritable nucléarisation semble s'être accru. Par ailleurs la nouvelle juxtaposition de l'hindouisme militant (de l'Inde) et du nationalisme islamique du Pakistan, crée les bases d'une telle mutation. Ses prémisses sont donc peut-être déjà présentes dans un changement des configurations régionales qu'affectent des données internes difficiles. A pondérer parce que très évolutives.

7 Un temps écarté par la mobilisation des forces pakistanaises sur un autre théâtre d'opérations, puis contenu par la volonté des gouvernements et la nouvelle diplomatie des deux pays, le vieux risque d'affrontements entre l'Inde et le Pakistan n'a pas disparu et un certain pourrissement de la situation du Pendjab et du Cachemire a donné à des acteurs extra-étatiques un rôle dont l'impact est d'autant plus imprévisible que la guerre, justement, a accusé la fracture des opinions.

La guerre du Golfe : un révélateur

8 La guerre a d'abord souligné les effets du désengagement américain au Pakistan. La présence militaire soviétique en Afghanistan avait valu à ce pays une importante aide militaire et une exceptionnelle tolérance des Etats-Unis devant un programme nucléaire dont l'importation clandestine de certains matériaux devait souligner l'ambiguïté. Après le retrait soviétique, les relations entre le général Zia et l'administration Reagan s'étaient détériorées par suite de l'avancement de ce programme (le Pakistan aurait expérimenté une bombe dès 1987) et du soutien, peu apprécié de Washington, accordé à des groupes afghans fondamentalistes. Enfin les protestations indiennes contre l'aide que les terroristes sikhs et les autonomistes cachemiris recevaient du Pakistan commençaient à bénéficier de quelque crédit.

9 A son arrivée au pouvoir, en décembre 1988, Benazir Bhutto, par contraste avec la politique étrangère "progressiste" qui avait été celle de son père Z.A. Bhutto, avait joué l'entente avec les États-Unis et une amélioration des relations avec l'Inde; elle avait aussi réaffirmé les buts civils de la recherche nucléaire. Cependant, pour se dégager de l'influence de l'armée, elle avait aussi rapproché le Pakistan des pays musulmans 
modernisateurs avant d'être renversée par le coup d'État d'août 1990 - qui avait durci les divisions de l'opinion.

10 Ensuite, le gouvernement islamiste de son principal adversaire, Nawaz Sharif, fut naturellement porté à soutenir la coalition anti-irakienne. Mais sur l'arrière-plan d'une certaine sympathie du Pakistan's People Party envers le rôle joué par Saddam Hussein et d'un essor du mouvement islamiste facilité par le projet gouvernemental d'inclure la Charia dans la loi fondamentale, une surenchère politique interne a réveillé un antiaméricanisme latent' et ouvert la voie aux grandes manifestations qu'a suscitées la guerre. Un certain mécontentement trouve maintenant son prolongement dans une contestation populaire sunnite de l'amitié avec l'Iran chiite. En Inde, l'autorisation de ravitaillement donnée aux avions américains par le gouvernement minoritaire de Chandra Shekhar a soulevé un tollé orchestré, en particulier par l'ancien Premier ministre V.P. Singh et, surtout, par Rajiv Gandhi qui, soutien décisif du gouvernement, en obtint l'annulation avant de mener sa propre offensive de paix en se rendant à Moscou et dans le Golfe, à la veille de l'offensive terrestre et du cessez-le-feu. Relevant surtout d'un pragmatisme à court terme, cette autorisation si contestée avait été donnée au moment où l'Inde souffrait de l'embargo anti-irakien qui amputait son commerce extérieur ( $6 \%$ avec les pays du Golfe) et la privait du pétrole irakien (représentant plus de $60 \%$ de son approvisionnement externe, par achats directs à l'Irak ou en exécution d'accords triangulaires avec l'URSS). Elle tenait compte de ses 600 millions de dollars de créances sur Bagdad et des risques encourus par ses entreprises de construction $\mathrm{du}$ fait $\mathrm{du}$ bombardement des Elle s'accordait enfin chantiers (installés, notamment, à Bassorah) avec le soutien de New Delhi aux votes du Conseil de Sécurité de l'O.N.U. sanctionnant l'Irak. La prudente politique de Chandra Shekhar procédait d'une certaine conception des intérêts nationaux. Mais, face aux pressions des partis (Janata Dal et, surtout, Congrès, qui cherchaient à cultiver l'électorat musulman, il qui le soutenait) fut contraint à un revirement peu propice à la solution de la crise financière(4) et, menacé de renversement, démissionna de son propre chef.

11 Tout en cherchant à tirer la leçon des bouleversements de l'ordre international dont ils perçoivent l'importance, les stratèges indiens sont, pour la plupart, incapables d'en prendre la pleine mesure car les réévaluations butent sur des certitudes idéologiques relatives au caractère essentiel du non-alignement et du rôle mondial de l'URSS. La prise de conscience d'une nouvelle autonomie des puissances moyennes renforce la crainte du fondamentalisme islamique, mais peu se défient d'États du proche horizon dont la puissance concurrence celle de l'Inde (car, après tout, c'est à l'armée indienne que l'armée irakienne, fût-ce seulement en paroles, a ravi sa consécration comme 4ème armée du monde). La part la plus visible de l'élite continue de voir le système international à travers le prisme usé d'une bipolarité dépassée. Ainsi la nouvelle de la mise au point, par l'Irak, d'un missile balistique de portée intermédiaire a seulement conduit les analystes indiens à penser que de nombreux pays dont, bientôt, le Pakistan, étant désormais dotés de ce type d'armes, il faudrait que l'Inde accélère son propre programme de missiles, même si ceux-ci ne devaient être dotés que de têtes conventionnelles(5).

12 Le seul tournant de la politique indienne aura finalement consisté à se situer dans une zone médiane d'accords, entre la position soviétique et la position française. Ensuite, remettant à l'ordre du jour le pacifisme dont elle se réclame et attisant les vieux 
réflexes Nord-Sud, la guerre a accusé les ambivalences d'une Inde qui est maintenant, à bien des titres, objectivement plus du Nord que du Sud et l'a amené à reformuler une politique plus traditionnelle. Pour le Pakistan, il s'agissait de gérer au mieux les ambivalences résultant de ses liens avec les Etats-Unis et du discours islamique. En discutant avec l'Iran et la Turquie des moyens de mettre fin à la guerre, Islamabad dévoilait les nouvelles tendances de sa politique étrangère: bien qu'engagé dans la coalition, un Pakistan qui avait perdu de son intérêt stratégique aux yeux des EtatsUnis jouait en même temps la carte islamique avec l'idée que le déclin de l'Irak lui donnerait une chance d'exercer un leadership.

L'Inde, quant à elle, réagit conformément à son rôle de pays-leader du Sud. Celui-ci passe par la reconnaissance que lui accorde le reste du monde musulman, au sein duquel elle occupe le deuxième rang, après l'Indonésie. Le soutien de l'Islam laïc, dont l'OLP, que Inde fut l'un des premiers grands pays à reconnaître, étant l'un des axes privilégiés des relations entre l'Inde et le monde arabe, il était naturel que New Delhi manifesta de la sympathie pour Bagdad. En outre, cause et effet de l'ambiguïté qu'elle a pratiquée, l'Inde a retrouvé en décembre un siège au Conseil de sécurité, ce qui explique sa concertation avec l'URSS et la Chine, pendant et après le conflit, comme ses efforts pour rendre un rôle aux non-alignés. La guerre du Golfe a aussi relancé en Inde certains débats stratégiques. Sous jacente à la tendance plus apparente à dénoncer la coalition comme un avatar de l'OTAN contribuant à déplacer la guerre froide, la réflexion sur la libéralisation économique et les moyens de la défense nationale est à l'ordre du jour avec, parfois, une nouvelle liberté dé ton concernant l'inadéquation de structures et projets spécifiquement indiens (tels que le Main Battle tank ou l'avion de combat léger) aux exigences technologiques et logistiques de la guerre moderne.

Rajiv Gandhi a lui-même fait rebondir les controverses sur le nucléaire et, devant une opinion déjà majoritairement acquise à la bombe, il a fait écho à l'argument, émanant de milieux militaires(6), selon lequel l'accession à la pleine capacité nucléaire serait la seule garantie possible de l'indépendance nationale. Le traité indo-pakistanais, de 1988, de "non-destruction" des installations nucléaires du voisin étant entré en vigueur en février 1990, cette prise de position pourrait se révéler lourde de conséquences. Car un nombre croissant d'experts indiens estime désormais qu'une paix garantie par l'équilibre de la terreur vaudrait mieux que l'insécurité actuelle et ce genre d'arguments rencontre un écho tout particulier dans les milieux de la droite nationaliste - dont, par ailleurs, les options de politique étrangère convergent davantage avec les anciens objectifs de l'OTAN. Compte tenu de la crainte de l'expansionnisme indien affichée par des pays comme le Bangladesh, Sri Lanka et le Népal, cette voie impliquerait, à termes, un risque de large prolifération sans garanties d'équilibre.

En contrepoint, et en opposition avec cette perspective, dessinée par le besoin indien d'opposer une force digne de ce nom à la puissance chinoise - que le traité sinosoviétique de 1989 a cessé d équilibrer par une protection soviétique implicite en faveur de Delhi - , une autre stratégie était dessinée en pointillé par les consultations sinoindiennes, que donnaient lieu à de nets progrès de la normalisation entre les deux pays (avec, notamment, la réouverture au commerce de postes-frontières). Particulièrement significatif au regard problème du Cachemire, ce tournant, en réunissant, sur des positions communes, deux pays qui représentent ensemble à peu près la moitié de l'humanité est lourd de menaces potentielles. En pesant sur les conditions mises par 
l'ONU au cessez-le-feu avec l'Irak, l'influence conjuguée (malgré les nuances nationales) de la Chine, de l'Inde et de l'Union soviétique est venue rappeler que, dans la confusion souvent faite entre non-alignement et esprit de Bandoeng, l'effacement du premier pouvait réveiller le second.

Inde - champ des possibles et nouvelles images

En outre, et sans qu'il soit possible de savoir dans quelle mesure les milieux militaires indiens (il n'y a pas en Inde de commandement suprême intégré pour l'ensemble des forces armées) se sentent engagés par une politique suggérée à un gouvernement Chandra Shekhar provisoire, une nouvelle option était offerte à l'Inde. En effet, non contents de se préparer à vendre à l'Inde des équipements électroniques sophistiqués qu'ils lui avaient longtemps refusés en raison de leur valeur militaire, les Etats-Unis, selon India today(7) l'auraient assurée non seulement d'une assistance dans la recherche de crédits et d'un rôle modérateur dans les relations indo-pakistanaises mais aussi d'encouragements à ce qu'elle devienne pleinement puissance tutélaire de la région (y compris dans l'océan Indien) grâce au développement d'une marine de haute mer. Ces discussions ont-elles joué un rôle dans la restitution à l'Union soviétique du sous-marin nucléaire qui lui avait été cédé en leasing ou le prix d'achat s'est-il simplement trouvé au-dessus des moyens actuels de l'Inde, comme la presse l'a suggéré ? La question reste posée. En tout cas, au cours d'une guerre soulignant le poids de certaines solidarités islamiques et les dangers de régimes militaires fuyant leurs problèmes dans une politique d'annexion l'Inde a, par comparaison avec le Pakistan, bénéficié d'une nette amélioration de son image.

17 Mais il n'est pas impossible que l'actuel rapport entre les diverses forces politiques indiennes y ait plus contribué qu'il n'y paraît. Car l'infléchissement de la politique étrangère alors enregistré en faveur de la coalition fut, en Inde même, porté au crédit (ou, plutôt, au discrédit) de Chandra Shekhar. Fait aussi symptomatique, lors des débats parlementaires houleux qui ont eu lieu sur les facilités consenties aux Américains, c'est en l'ancien ministre des Affaires étrangères du Janata, le dirigeant du BJP (parti du nationalisme hindou) A.B. Vajpayee, que Chandra Shekhar a trouvé son seul soutien d'envergure. Une fois la victoire de la coalition acquise, le BJP, qui avait jusque-là cultivé l'ambiguïté entre pacifisme et critique du soutien à Saddam Hussein, a clairement marqué sa réprobation d'une politique néfaste pour les intérêts indiens au Koweït. Il est vrai que, dans la presse, la guerre "anglo-américaine" était aussi devenue la victoire d'une authentique coalition associant des pays du Nord et des pays du Sud. Cependant les perceptions collectives ne semblent pas pour autant sensiblement modifiées.

18 Après avoir grossi les phénomènes, la guerre a donc aussi, au moins en Inde, provoqué un effet boomerang des retournements apparents Fournissant à Rajiv Gandhi l'argument critique d'une "rupture avec un non-alignement" qui forme la base ou consensus de politique étrangère, elle lui a donné les moyens d'ébranler, sans risques, un gouvernement qu'il soutenait à contrecoeur. Il est clair que les critiques de Rajiv Gandhi, provoquées par le mécontentement qui parcourait son parti, ont pris un ton d'autant plus acerbe que des élections étaient prévisibles à brève échéance. Néanmoins les pesanteurs sociologiques $\mathrm{du}$ parti du Congrès ont joué un rôle et, dans l'appréciation américaine des orientations futures de l'Inde, un facteur pourrait être plus déterminant qu'on le pense en France : le fait que le Congrès, donné vainqueur par les sondages (de 1991) ne paraisse plus certain d'obtenir une majorité absolue. C'était 
en effet à Rajiv Gandhi (comme à Indira Gandhi dans la période précédente, qu'étaient attribuées les politiques de puissance de l'Inde. Les intermèdes de gouvernements d'opposition ont tous, avec plus ou moins de succès, été marqués par des efforts d'apaisement.

La nouvelle importance de la droite incarnée par le nationalisme hindou(8) est aussi un changement de premier plan dont les effets peuvent être à double tranchant. L'existence même de l'éphémère gouvernement minoritaire de Chandra Shekhar tenait à la menace du Bharatiya Janata Party, à qui les sondages de fin 1989 donnaient un avantage considérable. Or, en tant que vecteur structuré d'un courant politique précis, le BJP est bien le parti dont l'anticommunisme et l'allergie à l'islamisme politique débouchent sur des réorientations stratégiques. La pénétration de ses idées et l'élan que lui a donné l'affaire de la "reconstruction" d'un temple hindou à la place de la mosquée édifiée, jadis, par Babar à Ayodhya (lieu, selon le mythe, de naissance de Rama) - notamment par le pèlerinage en char de L.K. Advani en 1990 - ne sont donc pas totalement étrangers au rapide et surprenant virage pris par le gouvernement indien. Le nouvel activisme des mouvements hindous est né d'une réaction contre les particularismes et contre les concessions des partis du centre (Congrès puis Janata Dal) aux autres forces "communalistes" (politiquement soudées par les appartenances communautaires), organisations sikhes et musulmanes en particulier; mais il engendre, en lui-même, une instabilité supérieure.

De tout ce qui précède, il ressort que l'avenir de la région sera marqué autant par une exacerbation des antagonismes culturels que par des choix délibérés. I1 sera également lié au rapport entre les niveaux de puissance et les espérances difficiles à satisfaire de multitudes plus proches de celles de l'Iran que de la population irakienne.

Diversification et intensification des menaces

21 La nouvelle menace de retombées des fermentations sociales: S'il fallait un exemple pour démontrer que l'une des principales menaces est à rechercher dans la contagion du fondamentalisme islamique, l'affaire Rushdie serait le plus parlant. En effet, on oublie trop qu'elle a trouvé son origine en Inde et que c'est par le réseau des solidarités islamistes que les versets sataniques ont été signalés à l'attention de dirigeants musulmans indiens puis du Pakistan et de l'Iran. Il est vrai que, en Inde et au Pakistan, le livre a seulement été interdit pour raisons d'ordre public.

Mais les risques liés à diverses formes de terrorisme et à l'impact des fondamentalismes ne s'arrêtent pas à ce type d'affaires. Bien que les mouvements indépendantistes sikhs adonnés à la violence aient récemment publié un code de conduite applicable à la presse (qui proscrit notamment, sous peine de représailles, l'emploi à leur sujet de l'épithète terroriste), et que le danger, si leurs aspirations demeurent insatisfaites, puisse être plus extensif et immédiat, comme l'ont montré certains attentats, ils ne seraient pas, par eux-mêmes, les plus déstabilisants. Plus graves, les liens entre trafics de drogue et terrorisme pourraient, en Asie comme en Amérique latine, devenir source d'oppositions Nord-Sud. Si corrupteur que puisse être le terrorisme des Tamouls de Sri Lanka (et son financement par les trafics de drogue, assuré par la diaspora qui le soutient), il n'a pas beaucoup inquiété jusqu'à ce qu'on lui impute l'assassinat de Rajiv Gandhi - ne serait-ce que parce que l'Inde, bénéficiant depuis 1987 de l'approbation tacite des grandes puissances, chargeait de maintenir sous contrôle les dangers inhérents au drame civil de l'île et que son gouvernement central, après une période d'ambivalence, tolère mal les camps rebelles du Tamilnadu. 
23 En revanche, les risques liés à une interconnection entre aide à différentes formes de subversion, trafics de drogue et phénomènes passionnels mobilisant la communauté musulmane peuvent d'autant moins être négligés qu'ils concernent directement le Pakistan, pays maîtrisant la technologie des missiles et possédant une réelle capacité de production nucléaire. Nés de l'aide du Pakistan aux insurgés afghans, ces trafics ont pris une extension difficile à contenir et les efforts en ce sens de Benazir Bhutto ont sans doute contribué à sa chute.

L'un des principaux griefs de l'Inde est du reste que, avec son assistance aux indépendantistes sikhs et cachemiris (ainsi que, depuis peu, aux insurgés de l'Assam à l'Est), le Pakistan entretient une contrebande qui, compte tenu des interpénétrations entre les deux pays, a inclus l'Inde dans le champ d'action de la mafia internationale. Jusqu'à présent, ni les Sikhs ni les Cachemiris ne paraissaient, en tant que tels, particulièrement liés aux mouvements de drogues. Mais, à côté des kidnappings imputables à des extrémistes cachemiris (la fille du nouveau ministre de l'Intérieur en novembre 1989 et, au début 1991, la fille d'un autre ministre), la presse indienne fait désormais état d'une dérive simplement délictuelle de la révolte de quelques groupes sikhs. Concernant le Pakistan, où des dirigeants de l'Établissement islamiste et militaire passent pour directement et profondément impliqués, cette menace commence à être prise au sérieux hors du sous-continent.

La menace qui pèse sur l'Inde, et pourrait faire tâche d'huile, est d'autant plus grave que, située entre le triangle d'or - dont la partie birmane entretient des relations avec les populations tribales du nord-est de l'Inde - et le Croissant d'or, l'Inde, pour certains, serait en passe de devenir une plaque tournante des trafics. Or la "criminalisation" d'une partie de sa classe politique observée depuis une dizaine d'années pourrait, dans un cadre sous-continental et à l'échelle du sixième de l'humanité, rendre plus difficile à policer que l'Amérique latine si le phénomène prenait l'importance qu'on lui prête au Pakistan.

Or les vagues de violences régionalistes (ou révolutionnaires, car il ne faut pas oublier que l'Andhra Pradesh est, de manière endémique, sujet à des actions naxalistes) sont désormais plus dévastatrices qu'autrefois parce que la centralisation des dernières décennies et l'essor des communications leur ont donné un impact direct sur les équilibres politiques nationaux qu'elles n'avaient pas sous Nehru. Ainsi, l'interconnection entre formes de protestations insurrectionnelles et criminalité pourrait, dans les hypothèses les plus pessimistes, assumer des proportions difficiles à imaginer. Mais il ne s'agit là que des aspects les plus voyants d'une montée de la violence entretenue par des inégalités croissantes entre villes et campagnes, entre bénéficiaires du développement et laissés pour compte, entre communautés qui s'attribuent l'essentiel des avantages et groupes rejetés aux marges. Le fait que la campagne pour les élections de mai 1991 se déroule en partie sur le thème des quotas d'emplois, postes électifs et places dans l'enseignement à réserver aux castes et groupes défavorisés et que V.P. Singh, principal champion de ces "réservations" soit allié aux partis communistes démontre une nouvelle dimension de lutte des classes dans la politisation, depuis longtemps observée, de forces sociales de plus en plus fragmentées et suggère la possibilité d'une vie politique affectée d'un mouvement brownien et faite d'inquiétudes renouvelées. A contrario, Chandra Shekhar n'a pas tort de souligner que les problèmes sociaux de l'Inde sont les mêmes depuis vingt ans et qu'il faut d'abord 
assurer la distribution d'eau potable dans les 600000 villages, combattre la pauvreté, la malnutrition et la maladie et que cela résoudra bien des problèmes politiques.

Laboratoire géant de tous les problèmes et acquis du siècle, une Inde capable de produire, si elle le décidait, une quinzaine au moins de bombes atomiques d'ici 1993, de produire en série ses propres missiles à courte et moyenne portée (expérimentés avec succès en 1989), lanceuse de satellites et partageant avec l'URSS son expérience en ce domaine pour la construction d'une commune navette spatiale, pourrait, par simple dérive, devenir le détonateur de conflagrations faisant passer la guerre du Golfe pour un incident. Des situations internes ingérables pourraient la conduire, de même que le Pakistan, à rechercher des succès extérieurs frappants. Mais elle n'a pas encore tourné le dos à la construction nationale identitaire tirée de la pédagogie nehruiste et, malgré le renouvellement des générations politiques, l'essentiel de ses dirigeants nationaux représente encore une élite responsable et pondérée consciente des retentissements internationaux de choix purement nationaux. De là à croire que certaines forces politiques sont, plus sûrement que d'autres, garantes de stabilité ou d'une consolidation des choix antérieurs, il y a un fossé qu'il serait aventureux de franchir. En effet, le parti du Congrès, qui a longtemps incarné la stabilité et la continuité, a eu sa part de responsabilités dans l'exacerbation des fondamentalismes : de la manière dont ses cadres, présents sur les lieux et, selon les témoignages plus bienveillants, passifs, se sont comportés au début novembre i984, au moment des pogroms antisikhs qui ont suivi l'assassinat d'Indira Gandhi à l'affaire des droits de la femme musulmane divorcée en 1985/86 ou à une conduite des affaires du Pendjab destinée à mobiliser l'électorat hindou de l'Haryana en 1987 - sans parler de la campagne électorale de 1989 les exemples ne manquent pas d'entorses du parti avec le sécularisme progressiste qui lui avait gagné l'estime des pays du Nord. Par contraste, la droite nationaliste du BJP, dont l'attitude assimilatrice à l'égard des Musulmans et l'opposition à toutes les formes de prosélytisme religieux (chrétien notamment) ne laissent pas d'effrayer le Nord offre, ou du moins, veut offrir une solution de rechange permettant de reconstituer un ciment national autour de valeurs stabilisatrices. Le succès que lui assure la désaffection envers les autres forces politiques se mesure à son ascension : parvenue à recueillir $12 \%$ des votes nationaux en 1989 (de 20 à 30\% dans certains États), elle est considérée comme ayant fait un bond considérable par ceux qui comparent le Bharatiya Janata Party de 1989 à ce qu'il était en 1980 et 1984 ou à ce qu'était le Jan Sangh de 1971, sans tenir compte de ce que la même force représentait au sein du Janata Party de 1977 (au moment où toutes les forces d'opposition non communistes s'étaient regroupées pour le constituer). En réalité, dès 1978, ses bastions du Madhya Pradesh ou de l'Himachal Pradesh représentaient des pourcentages de votes comparables à ceux de 1989, ce qui montre bien que des reflux sont possibles sur la base de problématiques nationales l'emportant sur les mobilisations passionnelles ou religieuses et que les inquiétudes qu'il suscite sont peut-être exagérées. Le BJP n'est pas certain d'une capitalisation politique durable sur les positions extrêmes qu'il a cultivées ces dernières années et le problème qu'il pose tient surtout à la légitimation d'attitudes d'exclusion engendrée par sa récente croissance.

Se posant maintenant comme hostile à la concentration et à la centralisation du pouvoir qui, selon lui, caractérisent la conception occidentale de l'État-nation (qui établit une suprématie de l'État sur la société opposée à la tradition hindoue), le BJP ne défend plus, comme il le faisait dans les années soixante, la conception d'un État unitaire et vise seulement, en principe, à une unité organique de la société. Mais cette 
unité, en soi, suppose le rejet de tout ce qui véhicule une culture étrangère communisme, socialisme, hiérarchie de valeurs judéo-chrétienne aussi bien qu'Islam ou sikhisme. Systématisé en refus d'imiter l'Occident par un courant de pensée qui déborde très largement la clientèle du mouvement hindouiste, ce rejet des apports étrangers (qui compense une ouverture et un attrait au moins égaux) prend une forme plus fruste au niveau populaire. Dans des phénomènes de foule, il se cristallise facilement en de violentes réactions xénophobes que les déploiements de police visent à prévenir au moment des fêtes religieuses. Plus généralement, ce nationalisme hindouiste, première incarnation du nationalisme indien née d'une réaffirmation, face à la domination coloniale, d'une civilisation de la qualité (au moins égale, sinon supérieure), millénaire, se nourrit de toute modernisation menaçante pour l'ordre traditionnel.

En caricaturant, on peut dire que, hors caste par définition, l'étranger (au système social hindou) est très bien admis et toléré s'il s'intègre et accepte la place qu'on lui consent. Qu'il revendique des droits, au nom de principes universels ou de rapports de forces, et il se voit à l'occasion opposer le bouclier d'une majorité culturelle prête à gommer les différences entre sectes et communautés en un rappel que les multiples voies de la spiritualité hindoue sont par essence supérieures au matérialisme du Nord ou à l'intolérance de l'Islam que l'universalisme peut, en lui-même, être l'ennemi, voire que l'Inde n'a que trop souffert de l'universalisme gandhien et nehruiste.

Indépendamment du potentiel émeutier qu'alimente la croissance de ce courant à l'intérieur même de l'Inde, il est facile d'imaginer les réactions qu'il déchaînerait en cas de crise internationale opposant l'Inde et les pays du Nord. Lorsqu'elle stigmatisait le rôle de donneurs de leçons des pays occidentaux, Indira Gandhi ne faisait que donner une expression laïque à ce profond courant nationaliste que, du même coup, elle détournait de la cible naturelle que constituent les partis réformateurs. Cependant, elle n'y est parvenue efficacement que tant qu'elle a pu satisfaire une aspiration latente aux démonstrations de puissance capables de laver deux siècles et demi d'humiliation et d'anéantir le vieux constat du Nord, toujours perçu comme une blessure, selon lequel la civilisation indienne est un système de stagnation, d'indifférence à la pauvreté, aux inégalités et aux malheurs des plus défavorisés.

Politique étrangère et mutations culturelles

31 Par contraste, la politique de puissance reprochée à Rajiv Gandhi a, en fait, été celle d'une puissance contenue, capable de satisfaire les exigences du nationalisme laïc qu'avait nourri Indira Gandhi et de répondre à l'attente d'une armée de plus en plus souvent employée à des tâches de pacification interne. Celle-ci trouvait dans son style et son niveau de vie comme dans les choix opérés pour sa modernisation de nombreux sujets de mécontentement. Dérivant souvent de l'excès de zèle des fonctionnaires, la politique de Rajiv Gandhi était un peu chaotique et plus inquiétante par la tonalité qu'y percevaient les pays voisins que par ses orientations délibérées. Car une certaine retenue l'a chaque fois marquée : les grandes manoeuvres militaires indiennes qui, en janvier 1987, ont failli déboucher sur une guerre indo-pakistanaise, parce que l'Inde n'en avait pas averti le Pakistan, se sont soldées par l'accord des deux pays pour ne pas attaquer les installations nucléaires de l'autre qui vient d'entrer en vigueur, la politique d'intervention à Sri Lanka de 1987 a pris fin en 1989, l'incursion aux Maldives de 1988 fut purement ponctuelle. En octobre 1987 l'Inde s'est abstenue d'intervenir au ?bet et sa manière de tenir la dragée haute au Népal, en 19881989, fut plus rigoureuse par le 
style que par le contenu car, paralysant effectivement le commerce népalais, elle n'a jamais, contrairement à ce qui fut dit, bloqué tous les postes frontières. L'aspect le plus inquiétant de la politique pratiquée sous Rajiv Gandhi tient aux coups de boutoir qui l'ont marquée car ils ont souvent semblé répondre à un besoin de légitimation.

Mais l'échec des gouvernements non-congressistes et les incertitudes du printemps 1991 devraient soulever d'autres questions. Les surenchères nationalistes sont, en fait, facilitées par l'émiettement des majorités et le seul moyen de dépasser l'opposition entre la régénérescence de l'Islam et l'hindouisme revanchard pourrait être de détourner les énergies collectives contre l'étranger. Cependant, rejoignant un courant congressiste, la pensée stratégique de certains, en ce qui est peut-être, à droite, un aggiornamento du vieux rêve d'effacer la partition, semble pousser à la conclusion d'un ensemble d'accords de sécurité collective dont l'existence ferait de l'Association Régionale de Coopération de l'Asie du Sud ou, au moins, du sous-continent, une espèce de confédération plus ou moins conforme à ce que les Etats-Unis avaient espéré promouvoir dans les années cinquante. Dans ce cas, la menace serait contenue.

A contrario, le schéma suivi à court terme va dans le sens d'un affrontement sur le Cachemire. En effet, tirant la leçon de ses récentes agitations, le BJP $a$, en son congrès de Jaipur de février 1991, choisi de privilégier sa revendication d'annuler les dispositions constitutionnelles garantissant l'autonomie de l'État au sein de l'Union indienne.

Dans ce cas, même le retour au pouvoir d'un gouvernement dirigé par le Congrès, s'il subissait une forte pression du nationalisme hindou, pourrait s'accompagner d'une activation de la dynamique de puissance. C'est une double vulnérabilité, politique et sociale, qui menace au premier chef la stabilité de l'Inde. Il en va de même au Pakistan et cette situation est entretenue par les fondamentalismes. Elle ouvre des perspectives d'autant plus dangereuses que l'Asie du Sud représente le cinquième de l'humanité.

Le vrai problème réside alors dans la capacité de nuire de nations dont les masses insatisfaites plébisciteraient des démagogues. Cette capacité parait supérieure au Pakistan si l'on tient compte de la réislamisation actuellement imposée et des à-coups qui ont ponctué son histoire. Le complexe du cadet qu'il cultive vis-à-vis de l'Inde, le fait que sa seule force politique inébranlable soit l'armée augmentent aussi les risques. Quels que soient les experts, tous les scénarios d'éventuelle conflagration liée à la prolifération nucléaire ont toujours attribué le rôle initiateur au Pakistan. Pour se comporter en grand frère, l'Inde, au moins, a jusqu'à présent montré une aptitude à rebondir qui autorise plus d'espoir.

Dans un cas comme dans l'autre, et c'est aussi vrai de pays moins puissants mais plus pauvres, comme le Bangladesh, on peut s'attendre à ce que le fondamentalisme, tous les particularismes et toutes les luttes sociales se nourrissent de l'insatisfaction de besoins dont le caractère subjectif est accru par la diffusion d'images télévisées et de filmsvidéo du monde entier. Ni l'Inde ni le Pakistan ne se voient menaçants pour autant que leurs aspirations à la justice soient prises en compte.

37 Toute la question posée au Nord, avec plus de nuances en ce qui concerne l'Inde, est donc de savoir s'il saura identifier sa propre part de responsabilité dans ce qu'il perçoit comme menace. 


\section{RÉSUMÉS}

Les menaces pouvant émaner de l'Asie du Sud ne sont plus enracinées dans le défi posé par le pouvoir croissant de l'Inde, leader du Sud, ou dans celui lié au risque d'un conflit local pouvant dégénérer en guerre mondiale. A la lumière des événements socio-politiques récents - avant et pendant la Guerre du Golfe - il semble que la principale menace réside dans la possible rupture de l'ordre international provenant de lois intérieures et de problèmes d'ordre (liés à l'augmentation de la vente illégale d'armes et de stupéfiants, dus au terrorisme international ou simplement dérivés d'une lutte politique féroce entre différents groupes sociaux). Bien que des changements dans la politique extérieure pakistanaise soient en cours et que l'Inde ne soit pas prête à un revirement de son approche du problème Nord-Sud, les événements s'étant déroulés durant la Guerre du Golfe ont surtout montré que les dérives naissaient de la surenchère entre pays faibles. Ainsi, l'intégrisme et le régionalisme croissants, nourris par des attentes grandissantes et déçues, menacent-ils la stabilité régionale et l'ordre mondial plus directement que le risque représenté par la poursuite d'une politique de militarisation contestant la suprématie du Nord.

Possible threats emanating from South Asia are no longer embedded in the challenge posed by the growing might of an India championing the South or by the risk of a regional war that could degenerate into a global one. In light of the recent socio-political developments - before and during the Gulf War - it seems that the main threat lies in the possible disruptions of international order stemming from internal law and order problems (related to increasing smuggling of arms and drugs, due to cross-boundary terrorism or simply derived from fiercer political competition between social communities). Though suggesting that changes in Pakistan's foreign policy are under way and proving that India is not ready for a reversal of its own approach to North-South problems, events that unfolded during the Gulf War mainly proved that drifts are induced by overbidding between weak parties. Thus, mounting fundamentalism and regionalism, nurtured by rising and unfulfilled expectations, menace regional and global stability more directly than the risk of pursuing conscious policies of military challenge to the Northern supremacy.

\section{INDEX}

Mots-clés : menace, risques

Index géographique : Asie du Sud-Est

\section{AUTEUR \\ CHRISTIANE HURTIG}

Chargée de recherches au CNRS, CERI 